\title{
ABNORMALITIES OF LIPOPROTEIN METABOLISM IN PATIENTS WITH THE NEPHROTIC SYNDROME
}

\author{
Jorge Joven, M.D., Carlos Villabona, M.D., Elisabet Vilella, Ph.D., Luis Masana, M.D., \\ Rosa Albertí, R.N.P., and Martin Vallés, M.D.
}

\begin{abstract}
Background and Methods. Patients with the nephrotic syndrome characteristically have multiple abnormalities of lipoprotein metabolism, but the cause and exact nature of these abnormalities are uncertain. In this study, we measured serum lipids and apoproteins in 57 patients with the nephrotic syndrome. We also determined the kinetic indexes of low-density lipoprotein (LDL) metabolism in six patients, and again in three of the six after recovery.

Results. The patients with the nephrotic syndrome had elevated serum concentrations of cholesterol, triglycerides, and phospholipids, which were confined to the lipoproteins containing apoprotein B. The serum concentrations of high-density lipoproteins and the associated $A-I$ and $A-I I$ apoproteins were similar in the patients with the nephrotic syndrome and normal subjects. The relative proportions of lipids and their positive association with
\end{abstract}

$\mathrm{H}^{2}$ YPERLIPIDEMIA is a striking feature of the nephrotic syndrome. It is a matter of debate, however, whether this hyperlipidemia leads to an increased risk of coronary heart disease and, if so, whether hypolipidemic therapy is indicated..$^{1-3}$ In addition, there are conflicting data regarding the distribution of cholesterol among the serum lipoproteins in patients with the nephrotic syndrome, ${ }^{4-7}$ and the different lipoprotein fractions have not been measured in a large group of such patients. This is probably because in most patients the nephrotic syndrome is accompanied by renal failure or other metabolic disorders such as diabetes mellitus (or both), or because the patients are receiving treatment, such as corticosteroids, that has a confounding effect on the lipoprotein patterns.

We attempted to determine prospectively the pattern of hyperlipoproteinemia in patients with untreated nephrotic syndrome who had normal renal function and no confounding metabolic disorders. In addition, because indirect evidence suggested that increased hepatic synthesis of low-density lipoprotein (LDL) was the primary cause of hypercholesterolemia in these patients, ${ }^{7-10}$ we carried out kinetic studies of LDL metabolism before and after recovery.

\section{Methods}

\section{Patient Selection}

We studied 57 patients with the nephrotic syndrome without complications and with no other illnesses. They were recruited from eight different hospitals over a three-year period. Their ages

From the Centre de Recerca Biomedica del Hospital de Sant Joan, Reus (J.J., C.V.); the Unitat de Recerca de Lipids, Reus (E.V., L.M.); the Hospital Germans Trias y Pujol, Badalona (R.A.); and the Hospital Valle de Hebrón, Barcelona (M.V.), all in Spain. Address reprint requests to Dr. Joven at the Hospital de Sant Joan, calle Sant Joan s/n, 43201 Reus, Spain.

Supported by grants from the Fondo de Investigaciones de la Seguridad Social and the Fundación Juan Abelló Pascual. the increased serum concentrations of apoproteins B, C-II, $\mathrm{C}-\mathrm{III}$, and $\mathrm{E}$ suggest quantitative rather than qualitative differences in the lipoproteins. All the patients had lipiduria, which probably reflected the excretion of highdensity lipoproteins, although no intact immunoreactive apoprotein A-I was found in urine. Serum albumin concentrations were inversely related to serum lipid concentrations in the patients, the severity of the hypoalbuminemia corresponding to the degree of change in serum lipoprotein concentrations. The kinetic studies of lipoprotein metabolism revealed an overproduction of LDL apoprotein B that returned to normal after recovery.

Conclusions. The elevated serum concentrations of LDL cholesterol, other lipids, and apoprotein B in patients with uncomplicated nephrotic syndrome are due to reversible increases in lipoprotein production. ( $N$ Engl $J$ Med 1990; 323:579-84.)

ranged from 15 to 81 years (mean [ $\pm \mathrm{SD}], 44.8 \pm 22.7$ ); 37 were male and 20 were female. All had serum creatinine levels of less than $110 \mu \mathrm{mol}$ per liter. They were selected from a group of 119 patients, among whom we excluded 39 because they had renal insufficiency, 10 because they had diabetes mellitus, and 13 because they had other disorders, including systemic lupus erythematosus, alcohol abuse, liver disease, obesity, familial hyperlipidemia, thyroid dysfunction, Cushing's syndrome, Addison's disease, and pheochromocytoma. None of the study patients were taking oral contraceptives, corticosteroids, cytotoxic drugs, or anticoagulant drugs, but 41 were taking furosemide in doses ranging from 40 to $120 \mathrm{mg}$ per day. As a group, these 41 patients had serum lipid and lipoprotein concentrations similar to those in the 16 patients not taking furosemide.

The nephrotic syndrome was defined as the excretion of more than $3.5 \mathrm{~g}$ of protein per day in the urine; serum albumin and total protein concentrations below 30 and $60 \mathrm{~g}$ per liter, respectively; and the presence of edema without other apparent cause. The mean ( $\pm \mathrm{SD}$ ) levels of the measured variables were as follows: serum creatinine, $86.7 \pm 21.4 \mu \mathrm{mol}$ per liter; serum albumin, $22.5 \pm 5.4 \mathrm{~g}$ per liter; serum total protein, $47.0 \pm 7.4 \mathrm{~g}$ per liter; and 24-hour urinary excretion of protein, $7.15 \pm 8.52 \mathrm{~g}$. Forty-five patients were studied when the diagnosis was first made, and 12 were studied at the time of a recurrence.

Percutaneous renal biopsies were performed in 55 patients. Twelve patients had minimal-change glomerulopathy, 18 membranous nephropathy, 7 membranoproliferative glomerulonephritis, 5 renal amyloidosis, 9 focal glomerulosclerosis, 3 mesangial proliferative glomerulonephritis, and 1 acute glomerulonephritis. None of the patients with renal amyloidosis had any preexisting or coexisting illness. We also studied 57 normal subjects ( 37 men and 20 women) as a control group. These subjects were matched with the patients for sex, age within five years, and weight within $5 \mathrm{~kg}$. None were taking any medications or had proteinuria, and all had normal renal function. The study was approved by the regional medical research committee, and all subjects gave informed consent.

\section{Kinetic Studies}

The rate of production and the fractional catabolic rate of LDL were studied in six patients when they had all the manifestations of the nephrotic syndrome and in three of the six after recovery. Their serum creatinine levels ranged from 75 to $89 \mu \mathrm{mol}$ per liter. Complete remission, defined as the absence of any clinical or laboratory manifestations of the nephrotic syndrome, was achieved spontaneously in two patients, whereas the third had received corticosteroid 
therapy for 45 days. The second kinetic study was undertaken two months after recovery and after all drugs had been discontinued.

For the LDL kinetic studies, $50 \mathrm{ml}$ of blood was drawn after an overnight fast, with $1 \mathrm{mg}$ of EDTA added per milliliter as an anticoagulant. LDL (density, 1.019 to $1.063 \mathrm{~kg}$ per liter) was isolated from plasma by preparative ultracentrifugation ${ }^{11}$ and labeled with iodine-125 (Amersham, Buckinghamshire, United Kingdom).$^{12,13}$ After dialysis for 24 hours with 25 liters of $0.15 \mathrm{M}$ sodium chloride and 0.01 percent EDTA (pH 7.5) to remove unreacted ${ }^{125} \mathrm{I}, 10 \mu \mathrm{Ci}$ of the autologous LDL was passed through a $22-\mu \mathrm{m}$ filter (Millipore, Velizy, France) and injected into the donor through an antecubital vein.

Ten-milliliter blood samples were collected from the contralateral arm 15 minutes after the injection of the ${ }^{125}$ I-labeled LDL and then daily for 10 to 14 days. Radioactivity was determined in $1-\mathrm{ml}$ aliquots and the counts expressed as a percentage of the count in the 15-minute sample. Plasma radioactivity-decay curves were constructed, and the fractional catabolic rate - defined as the fraction of total LDL apoprotein B removed per unit of time - was calculated from the biexponential curves according to the curve-peeling technique of Matthews. ${ }^{14}$ The rate of production of LDL apoprotein B, standardized for body weight, was calculated as the fractional catabolic rate times the size of the pool. The size of the pool was calculated as the serum LDL apoprotein B concentration times the volume of plasma. Serum $L D L$ apoprotein $B$ was measured with the 1,1,3,3-tetramethylurea-precipitation technique of Kane et al. ${ }^{15}$ The precipitate was extracted, dried in the presence of nitrogen, solubilized in $0.1 \mathrm{M}$ sodium hydroxide, and the protein assayed by the method of Lowry et al. ${ }^{16}$ For illustrative purposes, the results of the LDL kinetic studies in the patients with the nephrotic syndrome were compared with those in normal subjects and patients with hypercholesterolemia whom we had studied previously. ${ }^{17,18}$

\section{Other Studies}

The patients and normal subjects were studied after an overnight fast of at least 12 hours. The blood samples were centrifuged immediately after collection and the serum transported on ice to the Hospital de Sant Joan for analysis. The measurements were performed in fresh serum or in serum stored at $-20^{\circ} \mathrm{C}$, in which case the samples were analyzed in batches to minimize interassay variation. Twenty-four-hour urine samples were collected from all patients and normal subjects, sodium azide was added (final concentration, $2 \mathrm{mg}$ per liter) to prevent bacterial growth, and 50-ml aliquots were frozen for later analysis.

Total protein, albumin, creatinine, cholesterol, triglycerides, phospholipid, and apoproteins A-I, A-II, and B were measured in fresh serum by standard procedures, as was urinary protein in aliquots of 24-hour collections, in an autoanalyzer (2000 IL, Monarch, Milan, Italy). ${ }^{19}$ An aliquot of the serum was subjected to sequential preparative ultracentrifugation ${ }^{\prime \prime}$ to separate verylow-density lipoprotein (VLDL; density $<1.006 \mathrm{~kg}$ per liter) and LDL, and the tube was sliced to isolate the fractions quantitatively. High-density lipoprotein (HDL) was measured in the subnatant of the isolated LDL. Apoproteins C-II, C-III, and E were measured as previously described ${ }^{20}$ by single radial immunodiffusion on plates purchased from Daiichi Pure Chemicals (Tokyo, Japan). The interassay coefficient of variation for all measurements was less than 5 percent.

The urine samples underwent dialysis with phosphate-buffered saline, were concentrated 10-fold under pressure with use of an ultrafiltration membrane with a mean pore radius of $2.4 \mathrm{~nm}(24 \AA)$ (Amicon,
Danvers, Mass.), and then concentrated 25-fold in a standard filtration system (Millipore). Apoprotein A-I, cholesterol, triglycerides, and phospholipids were measured in the samples as described above. The limit of sensitivity for apoprotein A-I ranged from 15 to $20 \mu \mathrm{g}$ per liter. Sodium dodecyl sulfate-polyacrylamide-gel gradient (8 to 25 percent) electrophoresis was performed in prepared gels with use of the Phast System (Pharmacia, Uppsala, Sweden), and the resulting bands were identified by silver staining. The apparent molecular weight of each band was calculated from a calibration curve established with use of the low-molecular-weight calibrationkit proteins (Pharmacia). Trypsin was used as a control.

\section{Statistical Analysis}

The unpaired Student t-test was used to compare values in the patients with the nephrotic syndrome with those in the normal subjects and in the men and women, respectively. The paired Student t-test was used to compare the values in the patients with active nephrotic syndrome with the values in the same patients after recovery. $\mathbf{P}$ values of less than 0.05 were considered to indicate statistical significance. Pearson's correlation coefficients were calculated to evaluate the degree of linear association between measured variables.

\section{RESULts}

Nineteen patients (33.3 percent) had increased serum LDL and normal serum VLDL concentrations (Type IIa hyperlipoproteinemia, according to the World Health Organization's description of lipoprotein phenotypes $\left.{ }^{21}\right), 34$ (59.6 percent) had increased LDL and VLDL concentrations (Type IIb), and 4 (7 percent) had normal LDL and increased VLDL concentrations (Type IV). No patient had hyperchylomicronemia. The mean cholesterol, triglyceride, and phospholipid concentrations in serum and in the isolated lipoprotein fractions in all 57 patients are shown

Table 1. Lipid and Apoprotein Concentrations in Patients with the Nephrotic Syndrome and Matched Normal Subjects.

\begin{tabular}{|c|c|c|c|c|}
\hline VARIABLE & $\begin{array}{c}\text { MEN WITH THE } \\
\text { NEPHROTIC } \\
\text { SYNDROME } \\
(\mathrm{N}=37)\end{array}$ & $\begin{array}{c}\text { Normal } \\
\text { MEN } \\
(N=37)\end{array}$ & $\begin{array}{l}\text { WOMEN WITH THE } \\
\text { NEPHROTIC } \\
\text { SYNDROME } \\
(\mathrm{N}=20)\end{array}$ & $\begin{array}{c}\text { NORMAL } \\
\text { WOMEN } \\
(\mathrm{N}=20)\end{array}$ \\
\hline & \multicolumn{4}{|c|}{ mean $\pm S D$} \\
\hline \multicolumn{5}{|l|}{ Serum } \\
\hline Cholesterol (mmol/liter) & $9.35 \pm 3.24 *$ & $5.38 \pm 1.08$ & $9.38 \pm 3.50^{*}$ & $5.25 \pm 0.67$ \\
\hline Triglycerides (mmol/liter) & $2.60 \pm 1.20^{*}$ & $1.28 \pm 0.60$ & $2.52 \pm 1.39 *$ & $1.11 \pm 0.49$ \\
\hline Phospholipids (mmol/liter) & $4.24 \pm 1.14^{*}$ & $2.75 \pm 0.54$ & $4.42 \pm 1.57^{*}$ & $2.70 \pm 0.40$ \\
\hline Apoprotein A-I (mg/liter) & $1297 \pm 348$ & $1195 \pm 186$ & $1369 \pm 382$ & $1227 \pm 243$ \\
\hline Apoprotein A-II (mg/liter) & $298 \pm 60$ & $319 \pm 74$ & $335 \pm 58$ & $380 \pm 76$ \\
\hline Apoprotein B (mg/liter) & $1791 \pm 444^{*}$ & $833 \pm 210$ & $1766 \pm 468 *$ & $948 \pm 265$ \\
\hline Apoprotein C-II (mg/liter) & $66 \pm 25 t$ & $45 \pm 15$ & $77 \pm 29 *$ & $42 \pm 9$ \\
\hline Apoprotein C-III (mg/liter) & $168 \pm 72 *$ & $99 \pm 48$ & $199 \pm 85^{*}$ & $95 \pm 33$ \\
\hline Apoprotein E (mg/liter) & $89 \pm 52 \dagger$ & $68 \pm 35$ & $90 \pm 29 *$ & $45 \pm 9$ \\
\hline \multicolumn{5}{|l|}{ VLDL (mmol/liter) } \\
\hline Cholesterol & $1.21 \pm 0.72 *$ & $0.38 \pm 0.24$ & $1.13 \pm 0.90 *$ & $0.22 \pm 0.18$ \\
\hline Triglycerides & $1.35 \pm 0.90 \dagger$ & $0.65 \pm 0.42$ & $1.32 \pm 0.99 \dagger$ & $0.46 \pm 0.31$ \\
\hline Phospholipids & $0.68 \pm 0.36 *$ & $0.27 \pm 0.30$ & $0.61 \pm 0.42 *$ & $0.15 \pm 0.13$ \\
\hline \multicolumn{5}{|l|}{ LDL (mmol/liter) } \\
\hline Cholesterol & $6.36 \pm 2.70^{*}$ & $3.56 \pm 0.84$ & $6.42 \pm 2.90^{*}$ & $3.41 \pm 0.81$ \\
\hline Triglycerides & $0.96 \pm 0.40^{*}$ & $0.43 \pm 0.24$ & $0.93 \pm 0.40^{*}$ & $0.39 \pm 0.20$ \\
\hline Phospholipids & $2.22 \pm 0.95^{*}$ & $1.13 \pm 0.30$ & $2.34 \pm 1.05^{*}$ & $1.15 \pm 0.36$ \\
\hline \multicolumn{5}{|l|}{ HDL (mmol/liter) } \\
\hline Cholesterol & $1.77 \pm 0.60 \ddagger$ & $1.43 \pm 0.30$ & $1.82 \pm 0.74$ & $1.67 \pm 0.35$ \\
\hline Triglycerides & $0.27 \pm 0.15$ & $0.20 \pm 0.06$ & $0.27 \pm 0.15$ & $0.24 \pm 0.22$ \\
\hline Phospholipids & $1.35 \pm 0.30$ & $1.34 \pm 0.24$ & $1.47 \pm 0.37$ & $1.40 \pm 0.20$ \\
\hline
\end{tabular}

${ }^{*} \mathrm{P}<0.001$ for the comparison with the respective normal subjects. $+\mathrm{P}<0.01$ for the comparison with the respective normal subjects. $\ddagger \mathrm{P}<0.05$ for the comparison with the respective normal subjects. 


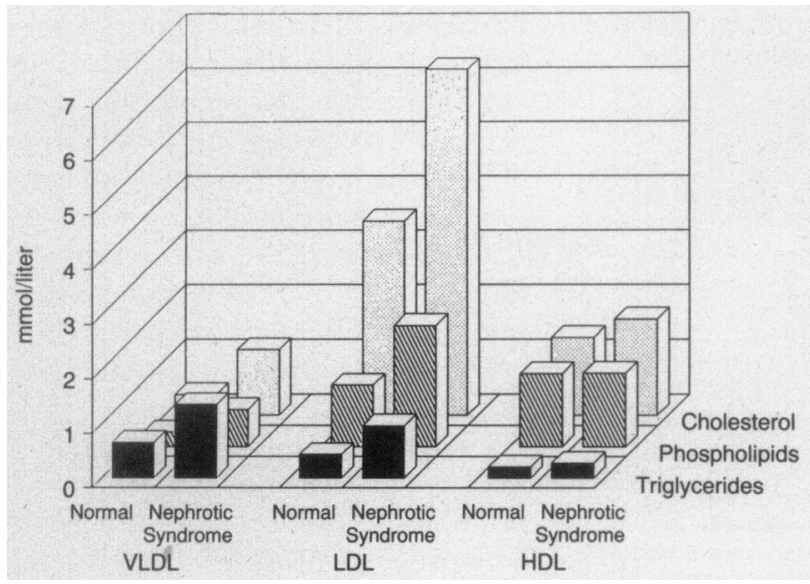

Figure 1. Mean Cholesterol, Triglyceride, and Phospholipid Distribution in the Lipoprotein Fractions of Serum of Normal Subjects and Patients with the Nephrotic Syndrome.

in Table 1 and Figure 1. Although no patient had normal levels of both cholesterol and triglycerides, the range of values was wide (cholesterol, 4.8 to 20.4 mmol per liter; and triglyceride, 0.9 to $5.0 \mathrm{mmol}$ per liter). The mean values for all three lipid components in serum and in the LDL and VLDL fractions were significantly higher in men and women with the nephrotic syndrome than in normal men and women. The increase in lipid components was greatest in the VLDL fraction (VLDL cholesterol, 3.9-fold increase; VLDL triglycerides, 2.5-fold; and VLDL phospholipids, 3.1-fold). In the LDL fraction, cholesterol was 1.8fold, triglyceride 2.3-fold, and phospholipid 2.0-fold higher in the patients with the nephrotic syndrome than in the normal subjects. In contrast, the concentrations of HDL lipids were only marginally elevated. There were no significant differences in the relative proportions of lipids in the lipoprotein fractions between the patients with the nephrotic syndrome and the normal subjects.

There were significant inverse linear correlations between the serum concentrations of albumin and cholesterol $(\mathrm{r}=-0.55 ; \mathrm{P}<0.001)$, triglycerides $(\mathrm{r}=-0.29 ; \mathrm{P}<0.05)$, phospholipids $(\mathrm{r}=-0.51$; $\mathrm{P}<0.001)$, and LDL cholesterol $(\mathrm{r}=-0.53 ; \mathrm{P}<0.01)$. There was no correlation between the 24-hour urinary excretion of protein and any of the lipoproteins measured.

Among the apoproteins, the mean serum apoprotein B, C-II, C-III, and E concentrations were higher in the patients with the nephrotic syndrome than in the normal subjects, for both men and women (Table 1). The largest percent increase was in serum apoprotein $B$ ( 115 percent in men and 86 percent in women), and the increase in apoprotein $B$ values correlated well with the increased LDL cholesterol values $(r=0.61$; $\mathrm{P}<0.001)$. The serum apoprotein A-I concentrations in the patients with the nephrotic syndrome (Table 1) were significantly correlated with their HDL cholesterol values $(r=0.68 ; \mathrm{P}<0.001)$. The serum apoprotein C-II and VLDL triglyceride levels $(r=0.26$;
$\mathrm{P}<0.05)$ and apoprotein $\mathrm{C}$-III and apoprotein $\mathrm{C}$-II levels $(r=0.79 ; \mathrm{P}<0.001)$ were also positively correlated in the patients with the nephrotic syndrome, and the ratio between apoproteins C-III and C-II was significantly higher $(\mathrm{P}<0.01)$ in the patients than in the normal subjects.

To determine whether the abnormal lipoprotein profiles bore any relation to the degree of hypoalbuminemia, we divided the patients arbitrarily into two groups - those with serum albumin levels of $21 \mathrm{~g}$ per liter or higher (18 men and 15 women; group 1), and those with levels below $21 \mathrm{~g}$ per liter ( 19 men and 5 women; group 2) (Table 2). The mean serum and LDL cholesterol, triglyceride, and phospholipid concentrations were significantly higher in group 2 than in group 1, as was the VLDL phospholipid concentration. Among the apoproteins, only serum apoprotein B levels were significantly higher in group 2 than in group 1. We found no relation between the histologic findings and any of the lipoprotein patterns.

All the patients but none of the normal subjects had lipiduria. The values for 24-hour excretion in the patients were as follows: cholesterol, 15.2 $\pm 10.8 \mu \mathrm{mol}$ per day (range, 1.2 to 36.1 ); triglycerides, $6.1 \pm 5.9$ $\mu \mathrm{mol}$ per day (range, 0.6 to 21.2 ); and phospholipids, $16.3 \pm 9.9 \mu \mathrm{mol}$ per day (range, 0.6 to 55.0 ). The relative proportions of cholesterol, triglycerides, and phospholipids (0.41:0.16:0.43) were similar to their proportions in the serum HDL fraction (0.37:0.08:0.55). This result prompted us to measure urinary apoprotein A-I. No immunoreactive apopro-

Table 2. Lipid and Apoprotein Concentrations in Patients with the Nephrotic Syndrome Grouped Arbitrarily According to Degree of Hypoalbuminemia.*

\begin{tabular}{|c|c|c|}
\hline VARIABLE & $\begin{array}{c}\text { GRouP 1 } \\
(\mathrm{N}=33)\end{array}$ & $\begin{array}{l}\text { Group } 2 \\
(N=24)\end{array}$ \\
\hline & \multicolumn{2}{|c|}{ mean $\pm S D$} \\
\hline \multicolumn{3}{|l|}{ Serum } \\
\hline Albumin (g/liter) & $25.7 \pm 2.9$ & $17.6 \pm 2.4 \dagger$ \\
\hline Cholesterol (mmol/liter) & $7.82 \pm 5.60$ & $10.90 \pm 7.11 \ddagger$ \\
\hline Triglycerides (mmol/liter) & $2.26 \pm 1.20$ & $3.01 \pm 1.22 \ddagger$ \\
\hline Phospholipids (mmol/liter) & $3.61 \pm 2.91$ & $5.01 \pm 4.50 \S$ \\
\hline Apoprotein A-I (mg/liter) & $1325 \pm 310$ & $1317 \pm 264$ \\
\hline Apoprotein A-II (mg/liter) & $243 \pm 69$ & $251 \pm 44$ \\
\hline Apoprotein B (mg/liter) & $1599 \pm 154$ & $2034 \pm 264 \dagger$ \\
\hline Apoprotein C-II (mg/liter) & $65 \pm 34$ & $77 \pm 29$ \\
\hline Apoprotein C-III (mg/liter) & $170 \pm 80$ & $191 \pm 83$ \\
\hline Apoprotein E (mg/liter) & $80 \pm 34$ & $69 \pm 34$ \\
\hline \multicolumn{3}{|l|}{ VLDL (mmol/liter) } \\
\hline Cholesterol & $1.05 \pm 2.92$ & $1.46 \pm 2.96$ \\
\hline Triglycerides & $1.14 \pm 3.84$ & $1.61 \pm 2.45$ \\
\hline Phospholipids & $0.55 \pm 0.91$ & $0.81 \pm 1.218$ \\
\hline \multicolumn{3}{|l|}{$\mathrm{LDL}$ (mmol/liter) } \\
\hline Cholesterol & $5.26 \pm 4.09$ & $7.38 \pm 6.40 \ddagger$ \\
\hline Triglycerides & $0.83 \pm 0.95$ & $1.10 \pm 1.14 \S$ \\
\hline Phospholipids & $1.85 \pm 1.02$ & $2.83 \pm 1.23 \ddagger$ \\
\hline \multicolumn{3}{|l|}{ HDL (mmol/liter) } \\
\hline Cholesterol & $1.67 \pm 2.67$ & $1.95 \pm 3.01$ \\
\hline Triglycerides & $0.26 \pm 0.45$ & $0.32 \pm 0.58$ \\
\hline Phospholipids & $1.40 \pm 0.93$ & $1.39 \pm 0.91$ \\
\hline
\end{tabular}

*The patients in group 1 had serum albumin levels $\geqslant 21 \mathrm{~g}$ per liter, and those in group 2 levels $<21 \mathrm{~g}$ per liter.

$+\mathrm{P}<0.001$ for the comparison with group $\mathrm{I}$

$\ddagger \mathrm{P}<0.01$ for the comparison with group $1 . \quad \S \mathrm{P}<0.05$ for the comparison with group 1 
Table 3. LDL Apoprotein B Kinetic Indexes in Patients with the Nephrotic Syndrome before and after Recovery, Patients with Two Types of Hypercholesterolemia, and Normal Subjects.*

\begin{tabular}{|c|c|c|c|c|}
\hline Group & $\begin{array}{c}\text { Fractional } \\
\text { CataBOLIC } \\
\text { RaTE }\end{array}$ & $\begin{array}{c}\text { RATE OF } \\
\text { PRODUCTION }\end{array}$ & $\begin{array}{l}\text { APOPROTEIN B } \\
\text { IN SERUM } \\
\text { LDL FrACTION }\end{array}$ & Pool Size \\
\hline & pools/day & $\mathrm{mg} / \mathrm{kg} /$ day & mg/liter & $m g$ \\
\hline $\begin{array}{l}\text { Patients with active nephrotic } \\
\text { syndrome }(n=6)\end{array}$ & $0.294 \pm 0.060$ & $15.25 \pm 6.51$ & $1092 \pm 400$ & $3126 \pm 1109$ \\
\hline $\begin{array}{l}\text { Patients after recovery } \\
\quad(\mathrm{n}=3)\end{array}$ & $0.283 \pm 0.058$ & $6.8 I \pm 4.18$ & $507 \pm 165$ & $1347 \pm 504$ \\
\hline Patients with $\mathrm{PH}(n=6)$ & $0.260 \pm 0.071$ & $10.50 \pm 1.83$ & $910 \pm 162$ & $2797 \pm 702$ \\
\hline Patients with $\mathrm{HFH}(\mathrm{n}=2)$ & $0.062 \pm 0.061$ & $13.69 \pm 1.75$ & $3312 \pm 1116$ & $2585 \pm 796$ \\
\hline Normal subjects $(n=7)$ & $0.330 \pm 0.092$ & $7.30 \pm 2.12$ & $501 \pm 190$ & $1453 \pm 220$ \\
\hline
\end{tabular}

*The previously reported results ${ }^{17,18}$ in the patients with polygenic hypercholesterolemia (PH) and the normal subjects, as well as those in the patients with homozygous familial hypercholesterolemia (HFH) studied for diagnostic purposes, are shown for comparison.

tThe fractional catabolic rate was defined as the fraction of total LDL apoprotein B removed per unit of time and is expressed as fractional pools per day.

tein A-I was detectable. When the urine was subjected to electrophoresis, however, 49 patients had a band corresponding to a molecular weight of approximately 27,000 . We did not identify this band as apoprotein A-I, but its size suggested that it was an apoprotein A-I-like protein.

The results of the kinetic studies of LDL metabolism are shown in Table 3 and Figure 2. The size of the LDL apoprotein B pool in the patients with the nephrotic syndrome was higher than that in the normal subjects. This increase was due to an increased rate of production; the fractional catabolic rate was similar to that of the normal subjects. The rate of LDL production in the patients with the nephrotic syndrome was higher than that in the two groups of patients with hypercholesterolemia. The fractional catabolic rate was similar in the patients with the nephrotic syndrome and those with polygenic hypercholesterolemia, whereas it was much lower in the patients with homozygous familial hypercholesterolemia. The serum lipid and apoprotein concentrations as well as the kinetic values were normal in the three patients studied after recovery.

\section{Discussion}

We measured the serum lipid concentrations and lipoprotein kinetics in carefully selected patients with the nephrotic syndrome who had normal glomerular function and no complicating diseases such as diabetes and who were not receiving any therapy other than furosemide. In agreement with previous studies, ${ }^{4-6,22,23}$ our patients had increased concentrations of cholesterol, triglycerides, and phospholipids in serum and in the VLDL and LDL fractions of serum, but the proportions of the individual components in the serum and lipoprotein fractions were similar to those in the normal subjects. These results, in combination with the increases in apoproteins, suggest that the abnormalities in serum lipoproteins in patients with the nephrotic syndrome are due to increased numbers of particles rather than abnormal particles, as have been described in children with the nephrotic syndrome. ${ }^{6}$ In contrast to the findings of Newmark et al., ${ }^{5}$ none of our patients had hyperchylomicronemia.

Although most investigators have found increased concentrations of VLDL and LDL in patients with the nephrotic syndrome, the results of HDL measurements have been contradictory, with high, ${ }^{22,24}$ low, ${ }^{25}$ and normal ${ }^{26,27}$ levels of HDL cholesterol being reported. The presence or absence'of chronic renal failure is a plausible explanation for this variation. Our patients had a selective increase in cholesterol in the apoprotein B-rich VLDL and LDL lipoprotein fractions. The mechanism of this selectivity is unclear, but it has been postulated that HDL cholesterol does not increase in these patients because it is lost in the urine. This possibility cannot be excluded in our study. However, we found no immunoreactive, intact apoprotein A-I in the urine of our patients, and the calculated 24-hour urinary losses of cholesterol, triglycerides, and phospholipids represented only $0.29,0.78$, and 0.39 percent, respectively, of the total circulating HDL lipids.

We found an absolute increase in serum apoproteins C-III and C-II in the patients with the nephrotic syndrome, together with an increased ratio of C-III to C-II consistent with a relative predominance of lipoprotein lipase inhibitor (apoprotein C-III) over activator (apoprotein C-II). These results, together with the previously reported decreases in lipoprotein lipase activity in patients with the nephrotic syndrome, ${ }^{28}$ suggest that VLDL catabolism is decreased in these patients. The finding of increased serum apoprotein $\mathrm{E}$ concentrations suggests an accumulation of apoprotein E-enriched particles of VLDL.

Increases in serum LDL concentrations, as found in patients with the nephrotic syndrome, could be due to overproduction, impaired catabolism, or both. Possible alterations in kinetic indexes are difficult to assess in case-control studies, because of the variations in such measurements and the near impossibility of achieving exact matching. These problems are obviated by serial studies conducted before and after recovery in individual patients. Our finding of an increased rate of production and normal fractional catabolic rate of LDL apoprotein B supports the work of oth$\mathrm{ers}^{10-29}$ indicating that overproduction of these lipoproteins is the chief cause of increased LDL concentrations in patients with the nephrotic syndrome. The normalization of serum LDL apoprotein B levels after recovery was due to diminished production; the fractional catabolic rate did not change. Different results were recently reported by Warwick et al., ${ }^{30}$ who found 

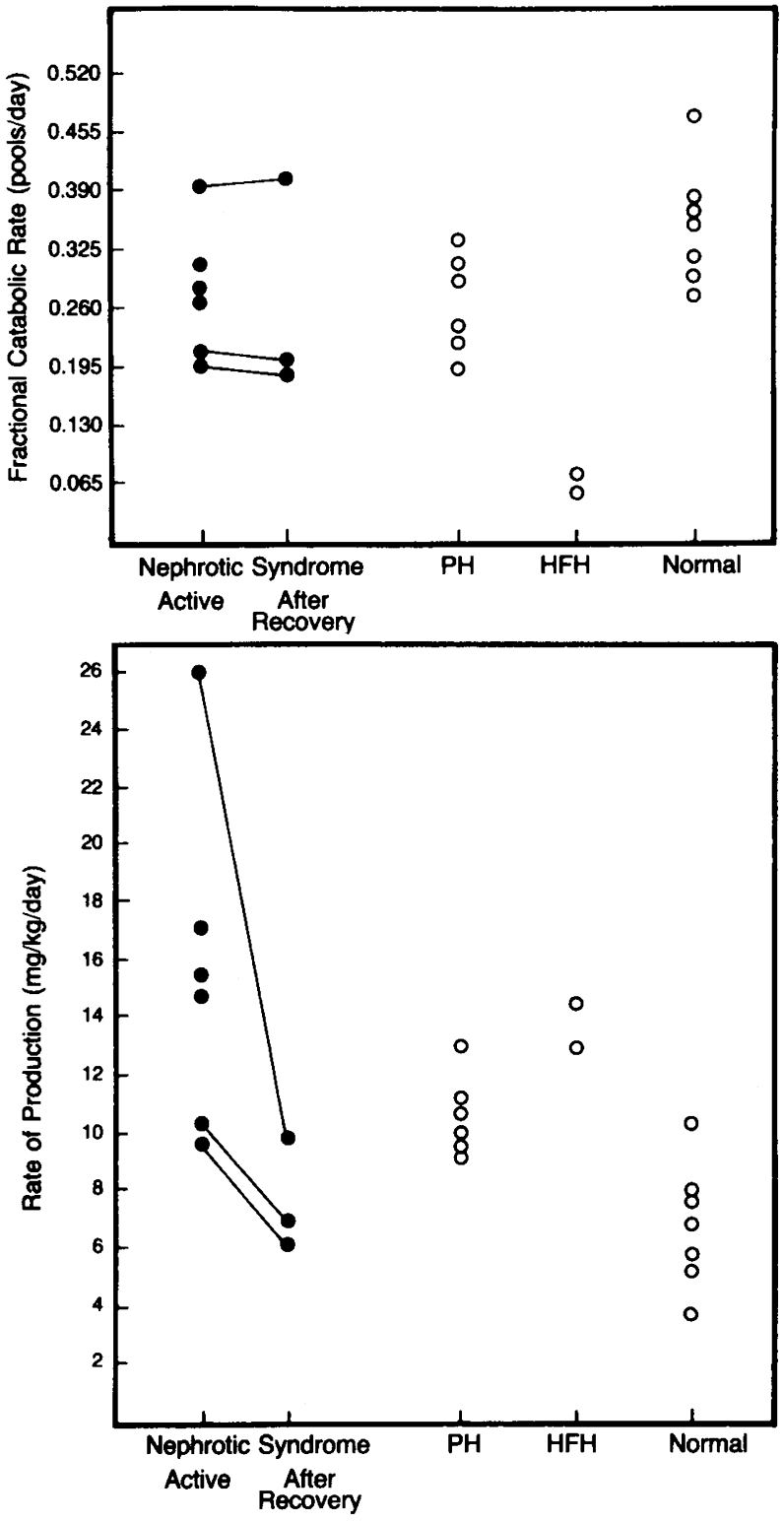

Figure 2. LDL Apoprotein B Fractional Catabolic Rate and the Rate of Production in Patients with the Nephrotic Syndrome (0) with Proteinuria and after Recovery.

For purposes of comparison, the results of previous studies $(O)$ in six patients with polygenic hypercholesterolemia $(\mathrm{PH}),{ }^{17}$ seven normal subjects, ${ }^{18}$ and two patients with homozygous familial hypercholesterolemia (HFH) studied for diagnostic purposes are shown.

that LDL apoprotein clearance by way of the LDLreceptor pathway was decreased. The subjects in the two studies were similar, although some of the patients of Warwick et al.$^{30}$ had mild renal insufficiency. Further studies are therefore needed to clarify this point.

The stimulus that triggers the increased production of apoprotein B is not clear. Hypoalbuminemia, low plasma oncotic pressure, and low plasma viscosity have been proposed as possible mechanisms. ${ }^{26,31}$ Our data support indirectly the pivotal role of hypoalbuminemia in the development of hyperlipoproteinemia. We hypothesize that decreased serum albumin levels are accompanied by a compensatory increase in LDL synthesis, particularly of the large, oncotically more effective apoprotein B. Further decreases in serum albumin may be accompanied by compensatory increases in other apoproteins that - particularly with respect to G-III and its inhibitory effect on lipoprotein lipase - could result in the accumulation of VLDL in the circulation.

In conclusion, patients with the nephrotic syndrome and hyperlipidemia appear to have a selective increase in LDL apoprotein B synthesis and normal catabolism of this lipoprotein. The normal serum levels of HDL and apoproteins A-I and A-II and the negligible urinary losses of apoprotein A-I make it unlikely that HDL metabolism is altered in patients with the nephrotic syndrome. These findings suggest that treatment directed toward inhibiting LDL synthesis (with the hydroxymethylglutaryl-coenzyme A reductase inhibitors, for example) would be appropriate.

We are indebted to the clinical and laboratory staffs of the Hospital Valle de Hebrón de Barcelona, Hospital Clínico y Provincial de Barcelona, Fundación Puigvert, Hospital de Bellvitge de Hospitalet de Llobregat, Hospital de San Andrés de Manresa, Hospital Germans Trias y Pujol de Badalona, Hospital de Sant Joan de Reus, and Hospital Juan XXIII de Tarragona.

\section{REFERENCES}

1. Wass VJ, Jarrett RJ, Chilvers C, Cameron JS. Does the nephrotic syndrome increase the risk of cardiovascular disease? Lancet $1979 ; 2: 664-7$

2. Mallick NP, Short CD. The nephrotic syndrome and ischaemic heart disease. Nephron 1981; 27:54-7.

3. Kannel WB, Castelli WP, Gordon T. Cholesterol in the prediction of atherosclerotic disease: new perspectives based on the Framingham study. Ann Intern Med 1979; 90:85-91.

4. Baxter JH, Goodman HC, Havel RJ. Serum lipid and lipoprotein alterations in nephrosis. J Clin Invest 1960; 39:455-65.

5. Newmark SR, Anderson CF, Donadio JV, Ellefson RD. Lipoprotein profiles in adult nephrotics. Mayo Clin Proc 1975; 50:359.

6. Gherardi E, Rota E, Calandra S, Genova R, Tamborino A. Relationship among the concentrations of serum lipoproteins and changes in their chemical composition in patients with untreated nephrotic syndrome. Eur J Clin Invest $1977 ; 7: 563-70$.

7. Golper TA, Swartz SH. Impaired renal mevalonate metabolism in nephrotic syndrome: a stimulus of increased hepatic cholesterogenesis independent of GFR and hypoalbuminemia. Metabolism 1982; 31:471-6.

8. McKenzie IF, Nestel PJ. Studies on the turnover of triglyceride and esterified cholesterol in subjects with the nephrotic syndrome. J Clin Invest 1968; 47:1685-95.

9. Joven J, Masana L, Villabona C, et al. Low density lipoprotein metabolism in rats with puromycin aminonucleoside-induced nephrotic syndrome. Metabolism 1989; 38:491-5.

10. Vega GL, Grundy SM. Lovastatin therapy in nephrotic hyperlipidemia effects on lipoprotein metabolism. Kidney Int 1988; 33:1160-8.

11. Havel RJ, Eder HA, Bragdon JM. The distribution and chemical composition of ultracentrifugally separated lipoproteins in human serum. J Clin Invest $1955 ; 34: 1345-53$.

12. McFarlane AS. The behaviour of $\mathrm{I}^{131}$-labelled plasma proteins in vivo. Ann N Y Acad Sci 1957; 70:19-25.

13. Langer T, Strober W, Levy RI. The metabolism of low density lipoprotein in familial type II hyperlipoproteinemia. J Clin Invest 1972; 51:1528-36.

14. Matthews CME. The theory of tracer experiments with ${ }^{131}$ I-labelled plasma proteins. Phys Med Biol 1957; 2:36-53.

15. Kane JP, Sata T, Hamilton RL, Havel RJ. Apoprotein composition of very low density lipoproteins of human serum. J Clin Invest 1975; 56:1622-34

16. Lowry OH, Rosebrough NJ, Farr AL, Randall RJ. Protein measurement with the Folin phenol reagent. J Biol Chem 1951; 193:265-75. 
17. Masana L, Escobar A, Joven J, et al. Treatment of diet-resistant polygenic hypercholesterolaemic patients with a new nicotinate derivative; in vivo and in vitro low density lipoprotein metabolic studies. J Clin Pharmacol 1989; 29:201-6.

18. International Collaborative Study Group. Metabolic epidemiology of plasma cholesterol: mechanisms of variation of plasma cholesterol within populations and between populations. Lancet 1986; 2:991-6.

19. Simo J, Bertran N, Juanpere M, Camps J, Joven J. Evaluación preliminar del analizador automático Monarch 2000. Quim Clin 1989; 8:329-35.

20. Joven J, Vilella E, Costa B, Turner PR, Richart C, Masana L. Concentrations of lipids and apolipoproteins in patients with clinically well-controlled insulin-dependent and non-insulin-dependent diabetes. Clin Chem 1989; 35:813-6.

21. Beaumont JL, Carlson LA, Cooper GR, Fejfar Z, Fredrickson DS, Strasser T. Classification of hyperlipidaemias and hyperlipoproteinaemias. Bull World Health Organ 1970; 43:891-915.

22. Ohta T, Matsuda I. Lipid and apolipoprotein levels in patients with nephrotic syndrome. Clin Chim Acta 1981; 117:133-43.

23. Chopra JS, Mallick NP. Hyperlipoproteinaemia in nephrotic syndrome. Lancet 1971; 1:317-20.

24. Jensen $\mathbf{H}$. Plasma protein and lipid pattern in the nephrotic syndrome. Acta Med Scand 1967: 182:465-73.
25. Alexander JH, Schapel GJ, Edwards KD. Increased incidence of coronary heart disease associated with combined elevation of serum triglyceride and cholesterol concentrations in the nephrotic syndrome in man. Med J Aust $1974 ; 2: 119-22$

26. Appel GB, Blum CB, Chien S, Kunis CL, Appel AS. The hyperlipidemia of the nephrotic syndrome: relation to plasma albumin concentration, oncotic pressure, and viscosity. N Engl J Med 1985; 312:1544-8.

27. Joven J, Rubiés-Prat J, Espinel E, Ras MR, Piera L. High-density lipoproteins in untreated idiopathic nephrotic syndrome without renal failure. Nephrol Dial Transplant 1987; 2:149-53.

28. Kashyap ML, Srivastava LS, Hynd BA, et al. Apolipoprotein CII and lipoprotein lipase in human nephrotic syndrome. Atherosclerosis 1980; 35:29-40.

29. Gitlin D, Cornwell DG, Nakasato D, Oncley JL, Hughes JL Jr, Janeway CA. Studies on the metabolism of plasma proteins in the nephrotic syndrome. II. The lipoproteins. J Clin Invest 1958; 37:172-84.

30. Warwick GL, Caslake MJ, Boulton-Jones JM, Dagen M, Packard CJ, Shepherd J. Low-density lipoprotein metabolism in the nephrotic syndrome. Metabolism 1990; 39:187-92.

31. Allen JC, Baxter JH, Goodman HC. Effects of dextran, polyvinylpyrrolidone and gamma globulin on the hyperlipidemia of experimental nephrosis. J Clin Invest 1961; 40:499-508.

\title{
SPECIAL ARTICLE
}

\section{THE LIFE EXPECTANCY OF PROFOUNDLY HANDICAPPED PEOPLE WITH MENTAL RETARDATION}

\author{
Righard K. Eyman, Ph.D., Herbert J. Grossman, M.D., Robert H. Ghaney, M.D., \\ and Thomas L. Gall, M.A.
}

\begin{abstract}
Background. The life expectancy of people with mental retardation is shorter than that of the general population. Exact estimates of the length of survival for mentally retarded persons at especially high risk are not available, however.

Methods. We collected data on mortality and other factors for 99,543 persons with developmental disabilities, including mental retardation, who received services from the California Department of Developmental Services between March 1984 and October 1987. Three subgroups were selected on the basis of the four characteristics identified in previous studies as the best predictors of mortality among mentally retarded people (deficits in cognitive function, limitations on mobility, incontinence, and inability to eat without assistance). In all three subgroups, the subjects had severe deficits in cognitive function and were incontinent; the subjects in subgroup $1(n=1550)$ were
\end{abstract}

$\mathrm{I}^{\mathrm{T}}$ Thas been well established that the life expectancy of children and adults with severe mental retardation is reduced as compared with that of the general population. ${ }^{1-4}$ However, no studies have specifically addressed the life expectancy of profoundly handicapped people who are mentally retarded - i.e., those who are unable to care for any of their personal needs.

From the Lanterman Developmental Center, University of California, Riverside (R.K.E., T.L.C.); the Departments of Pediatrics, Neurology, and Psychiatry, University of Michigan Medical School, Ann Arbor (H.J.G.); the School of Medicine, University of California, Los Angeles (R.H.C.); and the School of Medicine, Loma Linda University, Loma Linda, Calif. (R.H.C.). Address reprin requests to Dr. Eyman at the U.C. Riverside Research Group at Lanterman Developmental Center, P.O. Box 100-R, Pomona, CA 91769.

Supported in part by grants (HD22953 and HD21056) from the National Institute of Child Health and Human Development. immobile and required tube feeding; those in subgroup 2 $(n=4513)$ were immobile but could eat with assistance; those in subgroup 3 ( $n=997$ ) were mobile (but not ambulatory) and could eat with assistance. Life tables were generated for each of the three subgroups.

Results. Immobile subjects were found to have a much shorter life expectancy than those who could move about. Those who also required tube feeding (subgroup 1) had a very short life expectancy (i.e., four to five additional years). Those who could eat if fed by others (subgroup 2) had an average life expectancy of approximately eight additional years. In contrast, those who were mobile though not ambulatory (subgroup 3) had a life expectancy of about 23 additional years.

Conclusions. Severe mental retardation is associated with a decrease in life expectancy, particularly for those who are immobile. (N Engl J Med 1990; 323:584-9.)

The clinical practice of most physicians does not usually include treating mentally retarded persons, yet most handicapped children with mental retardation now survive the first years of life and live with their families in the community, where they will need medical treatment and guidance.

A number of previous studies have addressed the association of handicapping conditions with survival among mentally retarded people. ${ }^{2,3,5-20}$ The most common predictors of early death are the severity of retardation and deficits in mobility, toileting skills, and self-feeding. The presence or absence of seizures and of cerebral palsy does not generally add much additional predictive power. ${ }^{6}$ The best single predictor of early death is reduced mobility,,$^{3,6,7,10,12,16,20}$ followed 